NATIONAL LABORATORY

\title{
LCCP Desktop Application v1.0 Engineering Reference
}

\section{October 31, 2013}

\author{
Prepared by \\ Mohamed Beshr \\ Vikrant C. Aute
}

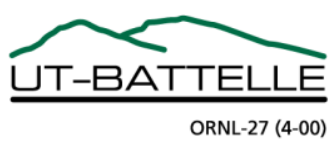





\title{
DOCUMENT AVAILABILITY
}

Reports produced after January 1, 1996, are generally available free via the U.S. Department of Energy (DOE) Information Bridge.

Web site http://www.osti.gov/bridge

Reports produced before January 1, 1996, may be purchased by members of the public from the following source.

\author{
National Technical Information Service \\ 5285 Port Royal Road \\ Springfield, VA 22161 \\ Telephone 703-605-6000 (1-800-553-6847) \\ TDD 703-487-4639 \\ Fax 703-605-6900 \\ E-mail info@ntis.gov \\ Web site http://www.ntis.gov/support/ordernowabout.htm
}

Reports are available to DOE employees, DOE contractors, Energy Technology Data Exchange (ETDE) representatives, and International Nuclear Information System (INIS) representatives from the following source.

Office of Scientific and Technical Information

P.O. Box 62

Oak Ridge, TN 37831

Telephone 865-576-8401

Fax 865-576-5728

E-mail reports@osti.gov

Web site http://www.osti.gov/contact.html

This report was prepared as an account of work sponsored by an agency of the United States Government. Neither the United States Government nor any agency thereof, nor any of their employees, makes any warranty, express or implied, or assumes any legal liability or responsibility for the accuracy, completeness, or usefulness of any information, apparatus, product, or process disclosed, or represents that its use would not infringe privately owned rights. Reference herein to any specific commercial product, process, or service by trade name, trademark, manufacturer, or otherwise, does not necessarily constitute or imply its endorsement, recommendation, or favoring by the United States Government or any agency thereof. The views and opinions of authors expressed herein do not necessarily state or reflect those of the United States Government or any agency thereof. 



\section{Table of Contents}

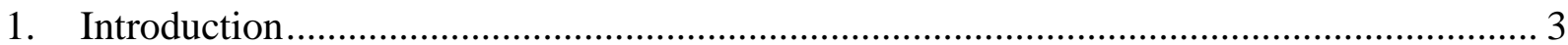

Part 1: Objectives, Literature Review and Mathematical Background..................................... 4

2. Overall Life Cycle Climate Performance Objective ...................................................... 4

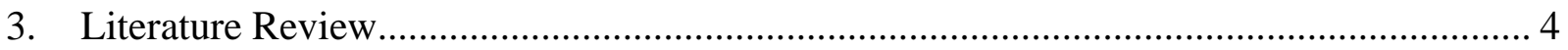

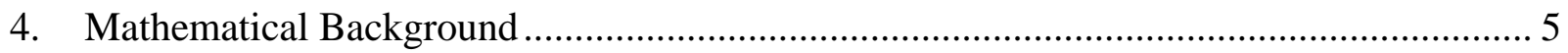

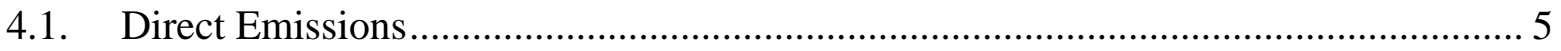

4.1.1. Emissions due to refrigerant leakage .................................................... 5

4.1.2. Irregular emissions due to such things as accidents .................................... 6

4.1.3. Emissions due to leakage of refrigerant during servicing ............................. 6

4.1.4. Emissions due to system end-of-life refrigerant loss ................................. 6

4.1.5. Emissions due to leakage from the refrigerant production and transportation ..... 7

4.1.6. Direct emissions ................................................................................. 7

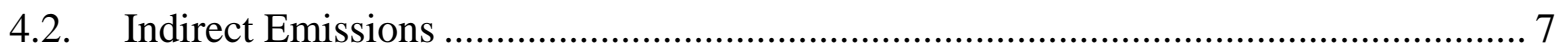

4.2.1. Emission due to energy used to manufacture components of system ................. 7

4.2.2. Emission due to energy used to manufacture refrigerant ................................. 8

4.2.3. Emission due to energy used to recycle end-of-life of components ................... 8

4.2.4. Emission due to energy used for end-of-life of refrigerants .......................... 9

4.2.5. Emission due to energy used to transport equipment ................................. 9

4.2.6. Emission due to energy consumption of system during system's lifetime .......... 9

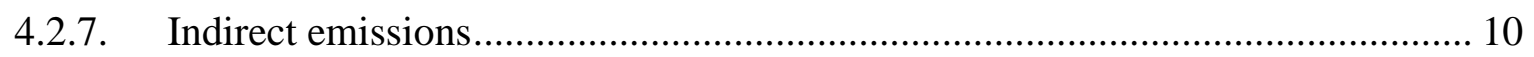

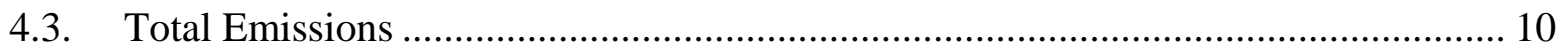

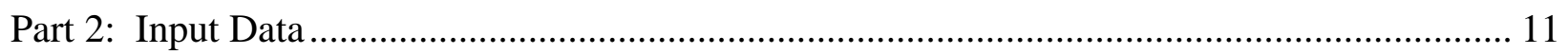

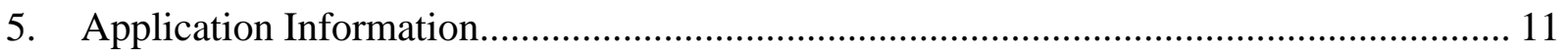




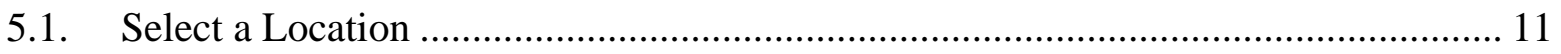

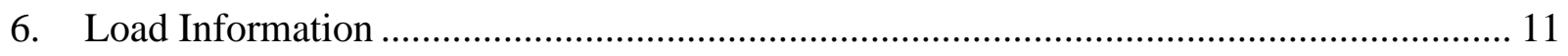

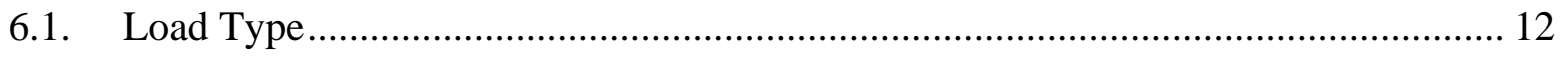

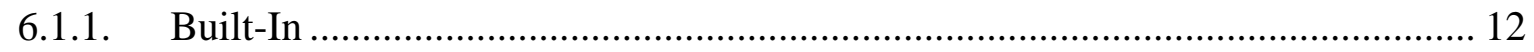

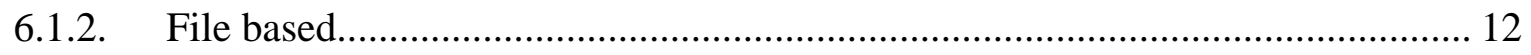

6.1.3. EnergyPlus ....................................................................................... 12

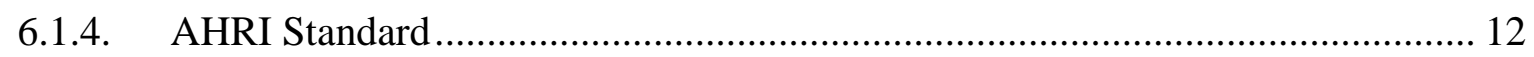

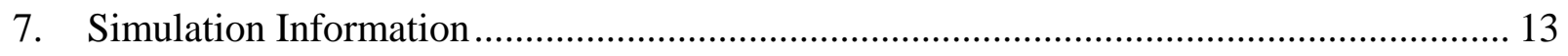

7.1. Centralized DX or Secondary Loop Systems ………………………….............. 13

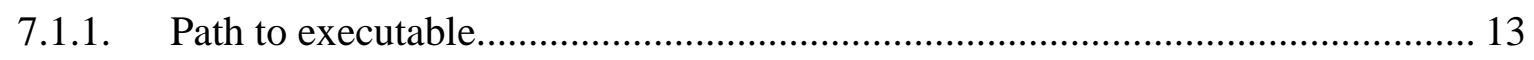

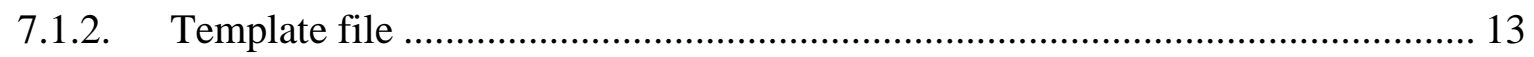

7.1.3. Working folder path ………………………............................................... 14

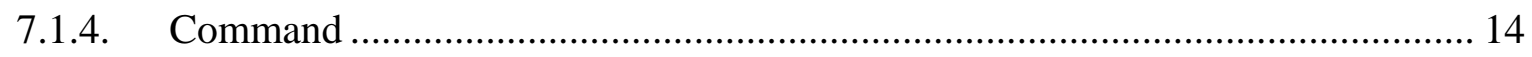

7.1.5. Account for charge degradation ...................................................................... 14

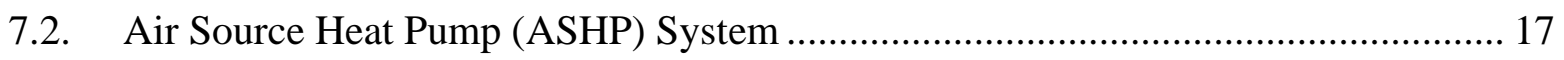

7.3. Display Cases Using Secondary Refrigerants ........................................................... 17

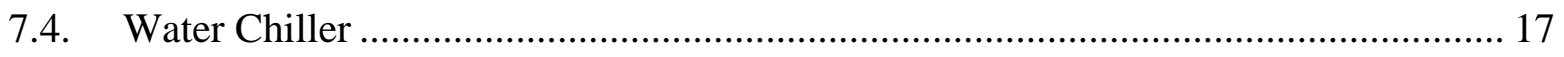

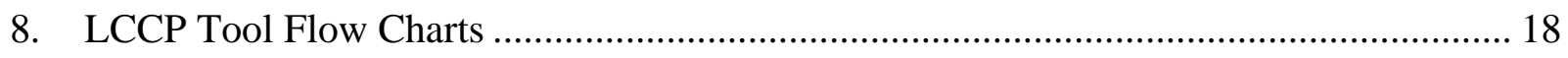

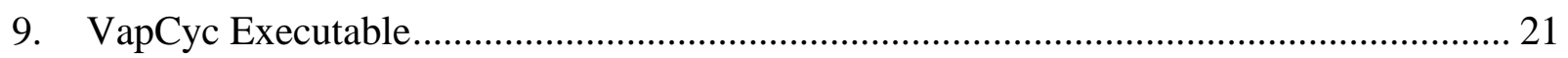

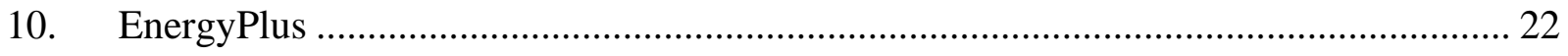

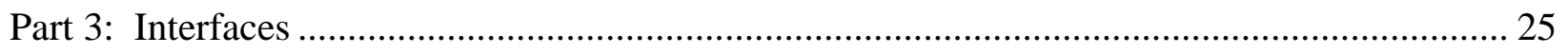

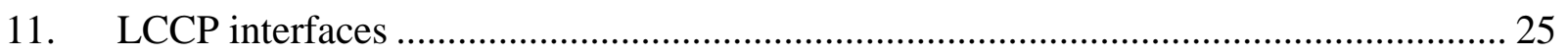

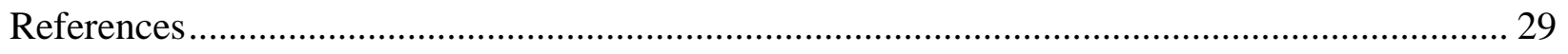




\section{LCCP Desktop Application v1.0 Engineering Reference}

\section{Introduction}

This Life Cycle Climate Performance (LCCP) Desktop Application Engineering Reference is divided into three parts. The first part of the guide, consisting of the LCCP objective, literature review, and mathematical background, is presented in Sections 2-4. The second part of the guide (given in Sections 5-10) provides a description of the input data required by the LCCP desktop application, including each of the input pages (Application Information, Load Information, and Simulation Information) and details for interfacing the LCCP Desktop Application with the VapCyc and EnergyPlus simulation programs. The third part of the guide (given in Section 11) describes the various interfaces of the LCCP code.

Figure 1 shows the main ORNL LCCP framework. The LCCP calculation methodology represents the core of the software. However, three main categories of inputs are required for these calculations. These categories are the emission and weather data (obtained from databases such as the TMY3 weather data), the load model (obtained from EnergyPlus, TRNSYS...etc.), and the system model (obtained from system performance models, or performance maps based on catalog data or experiments).

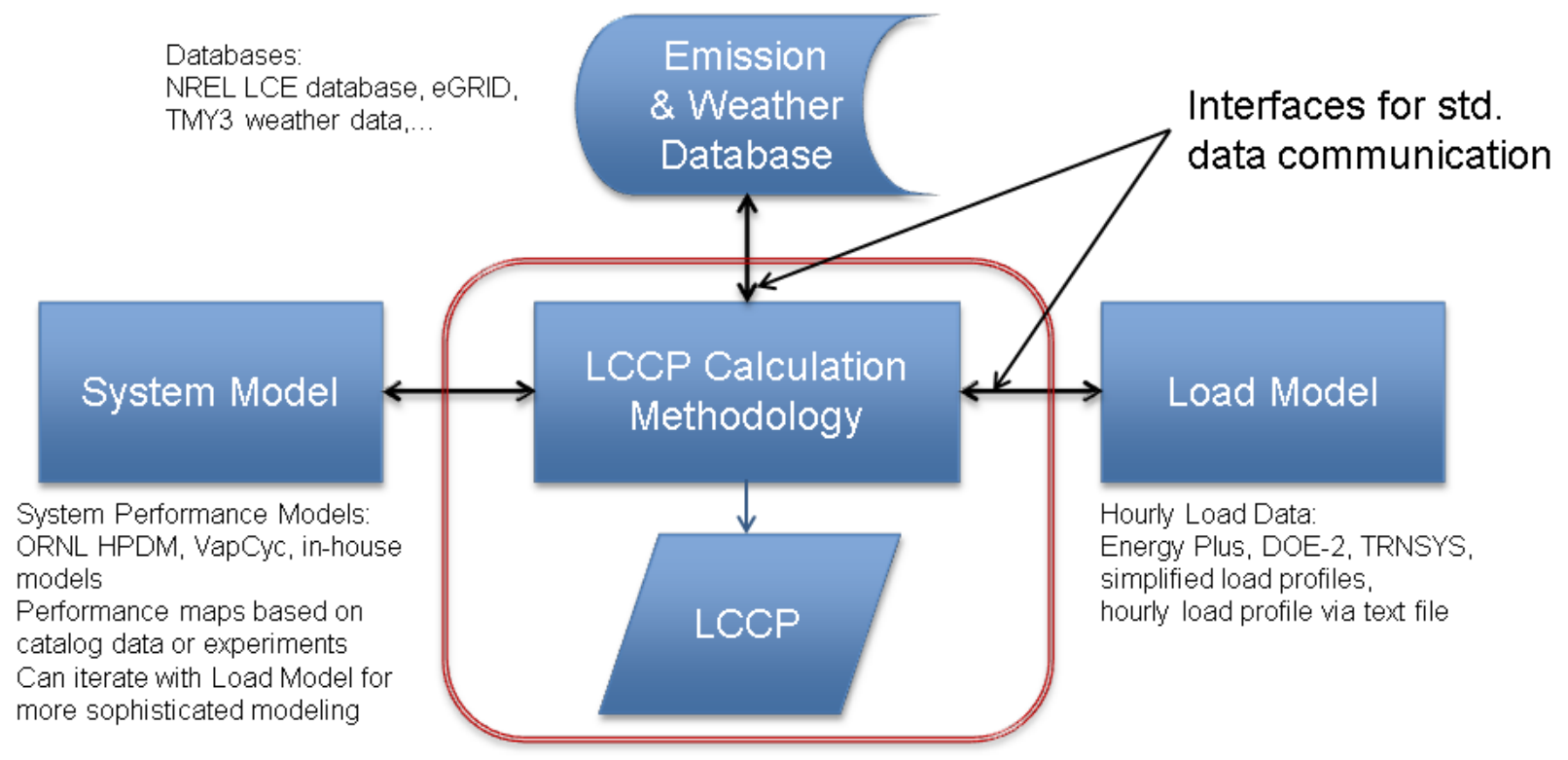

Figure 1: ORNL LCCP Framework. 


\section{Part 1: Objectives, Literature Review and Mathematical Background}

\section{Overall Life Cycle Climate Performance Objective}

The objective of Life Cycle Climate Performance (LCCP) analysis is to evaluate the equivalent mass of carbon dioxide released into the atmosphere due to a refrigeration system's performance, throughout its lifetime, from system construction through system operation and system destruction. The carbon dioxide emissions from a refrigeration system can be divided into two broad categories: direct emissions and indirect emissions. Direct emissions include the environmental impact of leakage of refrigerant which occurs during system operation. Indirect emissions include the environmental impact associated with the production and distribution of the energy required to operate the refrigeration system.

\section{Literature Review}

Sand et al. (1997) looked at the Total Equivalent Warming Impact (TEWI) of alternative refrigerants. They found insignificant decrease in TEWI in the use of ammonia and hydrocarbons as refrigerants for refrigeration systems with low emissions.

Papasavva et al. (2010) explained the workings of the GREEN-MACLCCP model in "GREENMAC-LCCP: A Tool for Assessing the Life Cycle Climate Performance of Mobile Air Conditioning (MAC) Systems.” In this paper they describe the methodology of the model as well as sample results generated from the model.

Deru and Torcellini (2007) detail the emission associated with energy usage in "Source Energy and Emission Factors for Energy Use in Buildings." In this report they detail the breakdown of the electricity grid in the United States as well as report the equivalent carbon dioxide emissions for electricity consumption in the different regions of the United States.

Hwang et al. (2007) experimentally tested walk-in refrigeration systems that simulate direct expansion commercial refrigeration systems with small charge utilizing R404A, R-410A, and R290 as refrigerants. They found that the LCCP of R-290A is always lower than that of R-404A. 
TMY3 data from the National Solar Radiation Data Base (NREL 2012a) details weather data for a number of different cities throughout the United States, including cities in such varied locations as Phoenix, Arizona, Los Angles, California, Chicago, Illinois, and New York, New York. The data sets include dry-bulb temperature (DBT), dew-point temperature, and relative humidity (RH) for all 8760 hours of the year.

The National Renewable Energy Laboratory maintains a Life Cycle Inventory database (NREL 2012b). Its goals include maintaining an accounting database that provides cradle-to-grave information about energy flows.

\section{Mathematical Background}

The following section provides the detailed mathematical procedure that is used in the LCCP Desktop Application for determining the carbon dioxide equivalent emissions of a system over its lifetime. The total emissions of a system are composed of direct emissions (due to direct leakage of refrigerant which occurs during system operation) and indirect emissions (due to the production and distribution of the energy associated with the construction, operation and decommissioning of the refrigeration system).

\subsection{Direct Emissions}

Papasavva et al. (2010) separate direct emissions into six categories, which are discussed below.

\subsubsection{Emissions due to refrigerant leakage}

Refrigeration systems oftentimes leak refrigerant during system operation. The direct emissions resulting from refrigerant leakage can be calculated as follows:

$$
\begin{aligned}
& \begin{array}{l}
\text { Emissions due to } \\
\text { refrigerant leakage }
\end{array} \\
& \text { System Charge * System Lifetime * Annual Leakage Rate * } \\
& \text { Global Warming Potential }(\mathrm{GWP}) \text { of Refrigerant }
\end{aligned}
$$

where System Charge (lb), System Lifetime (years), and Annual Leakage Rate (as a percentage of system the charge) are input by the user. 


\subsubsection{Irregular emissions due to such things as accidents}

Irregular emissions of refrigerant from the refrigeration system are due to unforeseen incidents and accidents. The direct emissions due to these accidents can be calculated as follows:

$$
\begin{gathered}
\text { Emissions due to accidents }=\text { System Charge } * \text { System Lifetime * Annual Accident } \\
\text { Emission Rate } * \text { GWP of Refrigerant }
\end{gathered}
$$

where System Charge (lb), System Lifetime (years), and Annual Accident Emission Rate (as a percentage of the system charge) are input by the user. If the user does not input a value for the Annual Accident Emission Rate, then the default value of zero will be used.

\subsubsection{Emissions due to leakage of refrigerant during servicing}

During refrigeration system servicing events, refrigerant may leak from the system. The direct emissions due to leakage of refrigerant resulting from servicing events can be calculated as follows:

$$
\begin{aligned}
\text { Emissions due to servicing }= & \text { Number of services in lifetime * System Charge } \\
& * \text { Servicing Leakage Rate * GWP of Refrigerant }
\end{aligned}
$$

where

Number of services in lifetime $=$ System Lifetime $/$ Service Interval;

and the System Charge (lb), System Lifetime (years), Service Interval, and Servicing Leakage Rate (as a percentage of the system charge) are input by the user. If the user does not input a value for the Servicing Leakage Rate, then the default value of zero will be used.

\subsubsection{Emissions due to system end-of-life refrigerant loss}

At the end of the useful life of the refrigeration system, the refrigerant in the system will be recovered prior to disassembly. During the refrigerant recovery process, a small quantity of refrigerant is inevitably released into the atmosphere. The direct emissions associated with the end-of-life loss of refrigerant can be calculated as follows:

Emissions due to end-of-life of system $=$ Percentage of Refrigerant Lost at End-of-Life $*$

$$
\text { System Charge * GWP of Refrigerant }
$$


where the System Charge (lb), and the Percentage of Refrigerant Lost at End-of-Life are input by the user.

\subsubsection{Emissions due to leakage from the refrigerant production and transportation}

When refrigerant is produced and transported to the location where it will be utilized, refrigerant leakage will occur. The direct emissions associated with refrigerant production and transportation losses can be calculated as follows:

Emissions due to production $=$ System Charge $*$ Refrigerant Production and Transportation and transportation $\quad$ Leakage Rate * GWP of Refrigerant

where the System Charge (lb) and the Refrigerant Production and Transportation Leakage Rate (as a percentage of the system charge) are input by the user. If the user does not input a value for the Refrigerant Production and Transportation Leakage Rate, then the default value of zero will be used.

\subsubsection{Direct emissions}

The aforementioned six contributors to the direct emissions may be combined to yield the total direct emissions as follows:

Direct Emissions = Emissions due to refrigerant leakage + Irregular emissions due to such things as accidents + Emissions due to leakage of refrigerant from servicing + Emissions due to end-of-life of system where refrigerant is lost + Emissions due to leakage that occurs from the refrigerant production and transportation + Reaction byproducts that occur from the atmospheric breakdown of the refrigerant emissions

\subsection{Indirect Emissions}

Papasavva et al. (2010) divide indirect emissions into six categories, which are discussed below.

\subsubsection{Emission due to energy used to manufacture components of system}

The indirect emissions associated with the energy that is used to manufacture the components of the refrigeration system is calculated as follows: 
Emission due to energy used to manufacture $=$ Mass of each material $*$

$$
\text { components of system } \quad \mathrm{CO}_{2} \text { equivalent }
$$

The $\mathrm{CO}_{2}$ equivalent term for each material is obtained from a database contained within the LCCP Desktop Application. The mass and material type of each component is specified by the user.

\subsubsection{Emission due to energy used to manufacture refrigerant}

The indirect emissions associated with the energy used to make the refrigerant itself is calculated as follows:

Emission due to energy used to $=$ System Charge $*(1+$ System Lifetime $*$ Annual Leak manufacture refrigerant Rate - Percentage of reused refrigerant) $* \mathrm{CO}_{2}$ equivalent emissions for virgin refrigerant

Equation (8) accounts for the refrigerant manufacturing emissions for the virgin refrigerant for all of the system charge, including the charge in the system and the charge leaked out of the system during the system's lifetime. The $\mathrm{CO}_{2}$ equivalent emissions for virgin refrigerant is obtained from a database contained within the LCCP Desktop Application.

\subsubsection{Emission due to energy used to recycle end-of-life of components}

The indirect emissions associated with the energy used to recycle the metal and plastic components of the refrigeration system at the end of the system's useful life is calculated as follows:

$$
\begin{aligned}
\begin{array}{c}
\text { Emission due to energy used for }= \\
\text { end-of-life components }
\end{array} & \text { Energy of Recycling of metals * mass of metals } * \mathrm{CO}_{2} \\
& \text { equivalent of metals }+ \text { Energy of Recycling of Plastics * } \\
& \text { mass of plastics* } \mathrm{CO}_{2} \text { equivalent of plastics }
\end{aligned}
$$

In equation (9), the energy required to recycle metals and plastics, and the carbon dioxide equivalent of metals and plastics is obtained from Papasavva et al. (2010). The masses of metals and plastics will be obtained from the refrigeration systems themselves, as specified by the user. 


\subsubsection{Emission due to energy used for end-of-life of refrigerants}

This factor quantifies the indirect emissions associated with the energy used to dispose of or recycle the refrigerants at the end of the useful life of the refrigeration system. The user enters a value for the total (throughout the life of the system) emissions due to refrigerant disposal or recycling (in $\mathrm{kgCO}_{2 \mathrm{eq}}$ ) as he or she sees fit. If the user does not enter a value for this factor, the default value of zero will be used.

\subsubsection{Emission due to energy used to transport equipment}

This factor quantifies the indirect emissions associated with the energy used to transport the refrigeration system from the manufacturing plant to the store location. The user enters a value for the total (throughout the life of the system) emissions due to energy used to transport the equipment (in $\mathrm{kgCO}_{2 \mathrm{eq}}$ ) as he or she sees fit. If the user does not enter a value for this factor, the default value of zero will be used.

\subsubsection{Emission due to energy consumption of system during system's lifetime}

This factor, which is probably the single biggest contributor to total emissions, involves the carbon dioxide equivalent emissions at the power plant related to the production and distribution of electrical energy that the refrigeration system utilizes. The indirect emissions associated with the energy consumption of the refrigeration system is calculated as follows:

Emission due to energy consumption $=$ System Lifetime $*$ Annual Energy Consumed $*$

of system during system's lifetime Average Emission Rate for Specified Location (10)

The Average Emission Rate for Specified Location is obtained from Deru and Torcellini (2007). To calculate the annual energy usage of the system, the following process will be used:

1) The ambient temperatures for all 8760 hours of a year will be obtained from Typical Meteorological Year (TMY) data. The TMY3 data will be obtained from the National Solar Radiation Database (NREL 2012a). In addition to taking temperature from all 8760 hours, binned data will be used to increase the speed of calculations.

2) These hourly ambient temperatures will then be used by VapCyc (or any other system simulation software) as the hourly environmental temperatures that the condenser is subjected to. 
3) The power output from VapCyc is the power consumed by the compressor. Summing the power over all 8760 hours in a year, or summing the binned power data, yields the Annual Energy Consumed, as used in Equation (11) above.

\subsubsection{Indirect emissions}

The aforementioned six contributors to the indirect emissions may be combined to yield the total indirect emissions as follows:

Indirect Emissions = Emission due to energy used to manufacture components of system + Emission due to energy used to manufacture refrigerant + Emission due to energy used for end of-life of components + Emission due to energy used for end-of-life of refrigerants + Emission due to energy consumption of system during system's

lifetime + Emission due to energy used to transport equipment (11)

\subsection{Total Emissions}

Finally, the total emission, which is the ultimate objective of the LCCP analysis and includes the contributions from direct and indirect sources, is given by:

Total Emissions $=$ Direct Emissions + Indirect Emissions 


\section{Part 2: Input Data}

The input data required by the LCCP Desktop Application is entered in three main pages: Application Information, Load Information, and Simulation Information. A description of each

of these input pages in addition to details for interfacing the LCCP Desktop Application with the VapCyc and EnergyPlus simulation programs is given in the following sections.

\section{Application Information}

This part of the inputs contains the input data which are used for the LCCP calculations (i.e. direct and indirect emissions). The different refrigerant leakage rates are input by the user and are used by the LCCP core to calculate the direct emissions and the indirect emissions except the indirect emissions due to the system's power consumption. The indirect emissions due to the system's power consumption are calculated based on the "Load Inputs" and "Simulation Inputs" for the code as explained in section 9, or based only on the "Load Inputs" as explained in section 10.

Also, this section contains the required information to obtain the hourly DBT, and RH. These values will be used together with the "Load Inputs" to create an input text file which will be placed in the working folder path (see section 7.3) for use by the system simulation software to calculate the system's hourly power consumption.

\subsection{Select a Location}

The user selects the location for which the LCCP calculations will be performed (i.e. the city where the analyzed system will be operated). Based on the location, the weather data, and the emissions values will be obtained from the TMY3 data files. The TMY3 files are found in the "AppFolder" folder in the installation directory of the LCCP desktop software. The "TMY3DataFiles" folder contains the weather data files while the "TMY3EmissionDataFiles" folder contains the emissions data. The files inside each folder follow the TMY3 nomenclature.

\section{Load Information}

The Load Information page contains the information required to specify the hourly absolute load values. These load values are used to create an input text file which is placed in the working 
folder path and provided to the system simulation software to calculate the system's hourly power consumption. Also, the system's hourly power consumption can be specified directly, as explained in section 10 .

\subsection{Load Type}

The user can select from three different load calculation methods:

\subsubsection{Built-In}

This option allows the user to specify the hourly load based on the nominal value (input by the user in the Application Information page), and a predefined hourly load profile (containing percentages of the nominal load).

\subsubsection{File based}

This option allows the hourly load to be specified via a user-supplied text file. The input file should be in a comma separated value format (.csv) with the load values being in the second column (and the first column can be the hour). This file can either contain the absolute load values in Watts (W) for every hour of the year or hourly values for the load as a percentage of the nominal load. Also, the file can contain the absolute power consumption values in Watt-hr (W-hr) for every hour of the year.

\subsubsection{EnergyPlus}

This option allows for the specification of the hourly load values through the use of a load calculation software (e.g. EnergyPlus). For this method, the user specifies the paths to the files required to run the load calculation software (see section 10 for more details). When the user selects this method, the option to either use EnergyPlus to obtain the hourly load values (and then run a system simulation software such as VapCyc) or directly obtain the system's hourly power consumption from EnergyPlus (without running a system simulation software) can be selected.

\subsubsection{AHRI Standard}

This option is only available when an air source heat pump (ASHP) system is selected. This option calculates the hourly load according to ANSI/AHRI Standard 210/240 (2008). Equations 
4.1-2 and 4.2-2 in the standard are used for the calculation of the cooling load, and heating load, respectively.

\section{Simulation Information}

In the Simulation Information page, the user specifies the data related to the system simulation. These data together with the load and weather data (specified in the Application Information page) are used to calculate the system's hourly and total annual power consumption. This part is required for the LCCP calculations unless the load type is file based (section 6.1.2) with absolute power consumption, or the calculation software (e.g. EnergyPlus) is used to directly obtain the system's hourly power consumption as explained in section 10.

\subsection{Centralized DX or Secondary Loop Systems}

For these two system types, a system simulation software is used to determine the hourly power consumption. The required inputs are explained in the following sections.

\subsubsection{Path to executable}

The user browses and selects the system simulation software (e.g. VapCyc) executable. This executable is supplied by the user and returns the "capacity" and "power consumption" of the system for every hour of the year (i.e. 8760 values of the system's capacity and power consumption). The output file should be in either a tab separated values format or a comma separated values (csv) format.

\subsubsection{Template file}

The user browses and selects the system simulation software input file template. This is simply the input file which will be read by the system simulation software in order to determine the capacity and power consumption. A sample template file for the VapCyc software is found in the "samples" folder in the "workingdirectory" folder located in the LCCP installation directory.

The template file can be modified by the user through the LCCP Simulation Information page. In order to do this, the user can replace any line in the template file (the line with the desired value change) with "Delimiter" + "Key" + "Delimiter" where "Delimiter" could be any symbol and "Key" could be any string. The user then writes a friendly name (any name or note for this parameter) in the LCCP user interface under the "Friendly Name" column. Also, the user writes 
the key (i.e. "Key") of the parameter in the "Key" column. Then, the user specifies the type of the parameter being changed under the "Type" column and its new or desired value under the "Value" column.

An example for this, based on the sample VapCyc template file, is replacing the condenser tube length with any desired value through the LCCP user interface. Also, the "Delimiter" is "\%\%" while the "Key" is "COND_TUBE_LENGTH". First, the condenser tube length line in the VapCyc file is replaced with the delimiter-key-delimiter string "\%\%COND_TUBE_LENGTH $\% \%$ " in order to create the VapCyc template file. Then, the key and the desired value are entered in the LCCP inputs page. When the user runs the LCCP software, the program searches for the "Key" in the template file and replaces it with the "value" specified by the user.

\subsubsection{Working folder path}

The user enters the path where the intermediate files will be saved. That is, this folder will have an input file which is passed from the LCCP core to the simulation software executable and contains the hourly weather data (DBT, and RH). The simulation software input file is simply the template file which results after replacing the key lines with the desired values. Finally, this folder will contain the output file written by the simulation software executable which will then be read by the LCCP core to perform the LCCP calculations.

\subsubsection{Command}

The user enters the command which contains the simulation software executable name and input arguments. For the VapCyc executable, this line has four inputs separated by a space. The first one is the name of the executable file (e.g. Executable.exe). The second term is the load file which is the input file to the system simulation tool and contains the hourly load and weather data. The third one is the template file which the system simulation software will run. The last term is the output file which is written by the executable and contains the system's hourly capacity and power consumption.

\subsubsection{Account for charge degradation}

The user can choose to account for the charge degradation on the system by checking the "Account for Charge Degradation". That is, the effect of refrigerant leak from the system, causing it to be undercharged, between two consecutive services on the performance of the 
system. When the user checks the "Account for Charge Degradation", a new window pops up. The user needs to enter the annual refrigerant slow leak rate, the charge limit after which the charge degradation would start to affect the system performance, the indoor DBT if it is required for the capacity and power consumption equations. In addition, the user needs to select the correlations for the effect of the charge degradation on the system's capacity and power consumption.

When running the cycle, the solver runs the system simulation tool just once and then performs the charge degradation calculations on the outputs. The charge decreases by an amount equal to the annual refrigerant slow leakage rate based on the nominal charge for every year (e.g. if charge is $100 \mathrm{lb}$ and leakage rate is $5 \%$, then after the first year the charge is 95 , and after the second year it is $90 \%$, and so on) until servicing is performed. This is repeated for the lifetime of the system.

A summary of some of the experimental studies done on the charge degradation effect on the system performance is summarized in Table 1.

Table 1: Summary of studies on effect of system undercharging.

\begin{tabular}{|l|l|l|}
\hline \multicolumn{1}{|c|}{ Study } & Year & \multicolumn{1}{|c|}{ System } \\
\hline The Trane company & 1976 & Eight year old residential air conditioner \\
\hline $\begin{array}{l}\text { A. A. Domingorena } \\
\text { Houcek and Thedford } \\
\text { for Texas Power and } \\
\text { Light (TP\&L) }\end{array}$ & 1980 & 3-ton air-to-air heat pump, R-22 refrigerant, heating mode \\
\hline $\begin{array}{l}\text { Mohsen Farzad and } \\
\text { Dennis L. O'Neal }\end{array}$ & 1988 & 3-ton Trane air conditioner with capillary tube expansion \\
\hline Mohsen Farzad & 1990 & 3-ton Trane air conditioner with three expansion devices \\
\hline
\end{tabular}




\begin{tabular}{|c|c|c|}
\hline Robinson & 1993 & $\begin{array}{l}\text { 3-ton split-system heat pump operating in the cooling mode. } \\
\text { The heat pump had a scroll compressor and utilized an orifice } \\
\text { for the expansion device. }\end{array}$ \\
\hline $\begin{array}{l}\text { Angel } \quad \text { Gerardo } \\
\text { Rodriguez }\end{array}$ & 1995 & $\begin{array}{l}\text { Two air conditioning units were used. One was a three ton, } \\
\text { split-system air conditioner with a scroll compressor and a } \\
\text { short tube orifice as the expansion device. The other unit tested } \\
\text { was a } 3.5 \text { ton, split system air conditioner with a scroll } \\
\text { compressor and a thermostatic expansion valve. }\end{array}$ \\
\hline $\begin{array}{l}\text { D.Y. Goswami, G. Ek, } \\
\text { M. Leung,C.K. Jotshi } \\
\text { and S.A. Sherif }\end{array}$ & 1997 & R-22 3-ton residential air-conditioning system \\
\hline J.M. Choi, Y.C. Kim & 2001 & $\begin{array}{l}\text { Water-to-water heat pump. The nominal cooling capacity of the } \\
\text { tested heat pump was } 3.5 \mathrm{~kW} \text {, and the working fluid was R } 22\end{array}$ \\
\hline $\begin{array}{l}\text { I.N. Grace, D. Datta, } \\
\text { S.A. Tassou }\end{array}$ & 2005 & $\begin{array}{l}\text { A small } 4 \mathrm{~kW} \text { nominal cooling capacity vapor compression } \\
\text { water-to-water chiller equipped with plate type heat exchangers }\end{array}$ \\
\hline $\begin{array}{l}\text { Erin Kruse and Larry } \\
\text { Palmiter }\end{array}$ & 2006 & $\begin{array}{l}\text { The heat pump tested was a 3-ton economy model with a rated } \\
\text { Seasonal Energy Efficiency Ratio (SEER) of } 10 \text { and a Heating } \\
\text { Seasonal Performance Factor (HSPF) of } 7.2 \text { (a 3-ton Carrier } \\
\text { YKC heat pump (R-22 refrigerant, suction-line accumulator) in } \\
\text { heating mode only). All of the measurements were performed } \\
\text { once with each of two metering devices: a short-tube orifice } \\
\text { (STO) and a thermostatic expansion valve (TXV) on the } \\
\text { outdoor unit. }\end{array}$ \\
\hline $\begin{array}{l}\text { Adam Wichman, } \\
\text { James E. Braun }\end{array}$ & 2008 & $\begin{array}{l}\text { The cooler system utilized R-22 as the refrigerant, whereas the } \\
\text { freezer unit employed R-404A. Both systems were equipped } \\
\text { with a TXV expansion valve and a liquid line receiver. }\end{array}$ \\
\hline
\end{tabular}




\begin{tabular}{|c|c|c|}
\hline $\begin{array}{l}\text { Woohyun Kim, James } \\
\text { E. Braun }\end{array}$ & 2010 & see tables $1-4$ in the paper \\
\hline $\begin{array}{l}\text { Bo Shen, James E. } \\
\text { Braun, Eckhard A. } \\
\text { Groll }\end{array}$ & 2011 & 3-ton R-22 split heat pump in heating mode \\
\hline
\end{tabular}

\subsection{Air Source Heat Pump (ASHP) System}

The hourly power consumption of the system is calculated according to the procedure presented in section 4 of the ANSI/AHRI Standard 210/240 (2008). The load type for an ASHP system is either EnergyPlus or AHRI standard.

\subsection{Display Cases Using Secondary Refrigerants}

The hourly power consumption of the system is calculated according to the procedure presented in section 5.2 of the ANSI/AHRI Standard 1320 (2011). There is no load input required for this system.

\subsection{Water Chiller}

The hourly power consumption of the system is calculated using the ANSI/AHRI Standard 550/590 (2011) according to the following procedure:

1) The user enters some information about the performance at the part load rating conditions specified in table 3 in the standard. That is, the user will enter the full capacity of the system and the EER at $25 \%, 50 \%$, and $75 \%$ load.

2) Based on this information and the hourly load profile, the hourly part load factor will be determined. Thus, the hourly EER will be calculated using linear interpolation between each of the user entered values (points). Thus, the hourly power consumption can be calculated. If the user doesn't have the data at the main part load factors $(25 \%, 50 \%$, $75 \%)$, the user can enter the data available and also linear interpolation will be applied between each of the entered points (refer to section 5.4.1.2 in the standard). However, up to this point the ambient temperature effect is not accounted for. 
3) The user would enter 2 more inputs. The first one is an equation for the EER as a function of ambient temperature and the part load factor. The second input would be the rating temperature at which the user did the experiments (i.e. testing ambient temperature). Based on these 2 inputs and the EER obtained in step 2, the ambient temperature adjusted EER will be calculated and hence the hourly power consumption will be calculated.

It is worth mentioning that there is no load input required for this system type.

\section{LCCP Tool Flow Charts}

The LCCP desktop software provides three different options for specifying the system's hourly load or power consumption. The first option is through obtaining the hourly load using the "Load Inputs" found on the Load Information page (i.e., built in, user input absolute load values file, user input load percentage file, Energy Plus software for load calculation). When using this option, the user must provide the "Simulation Inputs" (found on the Simulation Information page) that are required to run the system simulation software (e.g. VapCyc). The flow chart for this option is shown in Fig. 2 below. 


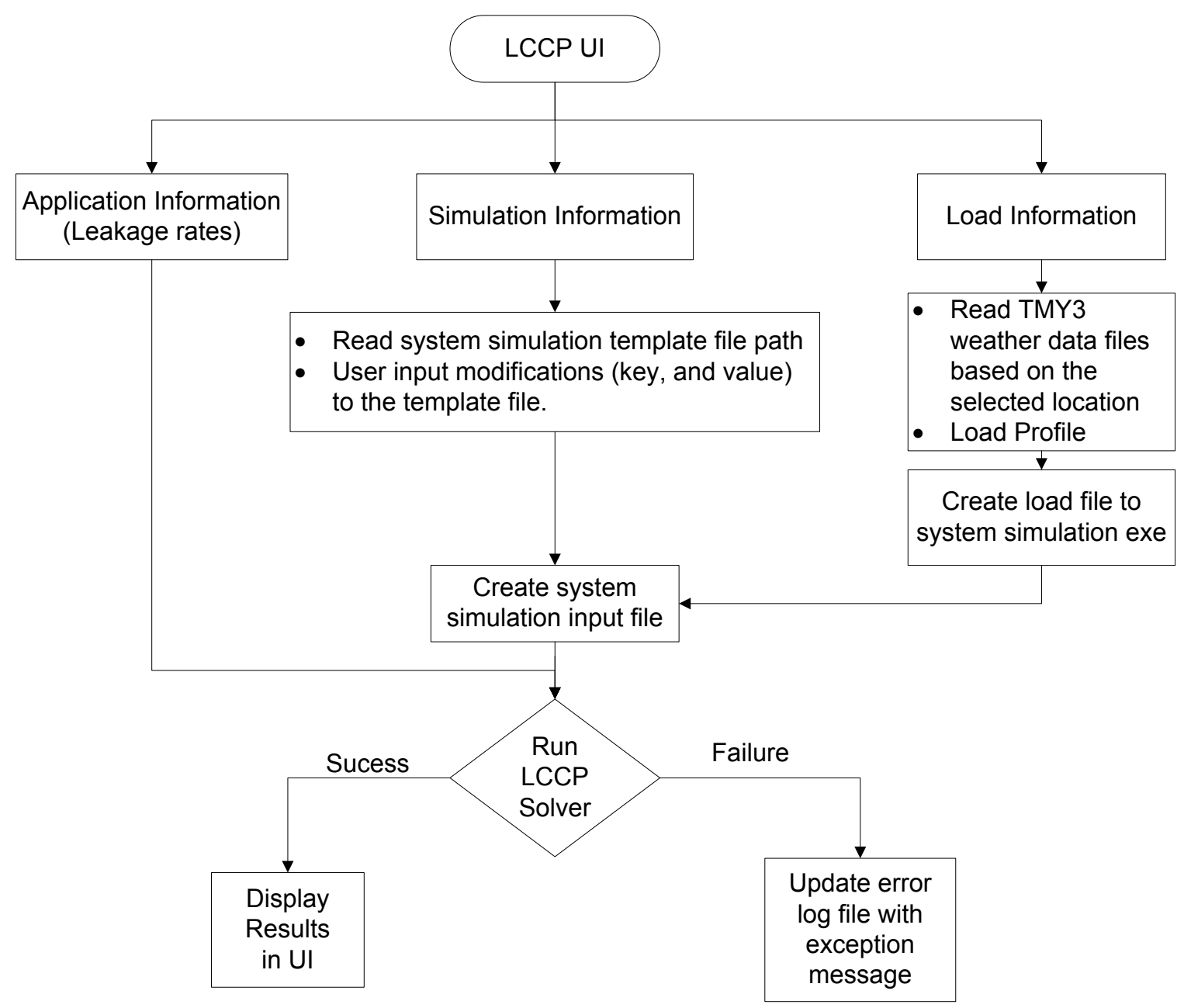

Figure 2: Flow Chart of the LCCP Desktop Application using all inputs.

The second option is to specify the hourly power consumption directly from the "Load Inputs" found on the Load Information page (i.e., selecting Energy Plus and then choosing Power Consumption) without requiring the "Simulation Inputs" from the Simulation Information page. In this case, the user will be asked to enter the path to the Energy Plus system layout file (.idf file), and the path to the Energy Plus weather data file (.epw file). Also, the user will need to enter the column header of the column containing the hourly power consumption values in the output (.csv) file (e.g. "Whole Building:Total Building Electric Demand [W](Hourly)"). The flow chart for this option is shown in Fig. 3 below. 


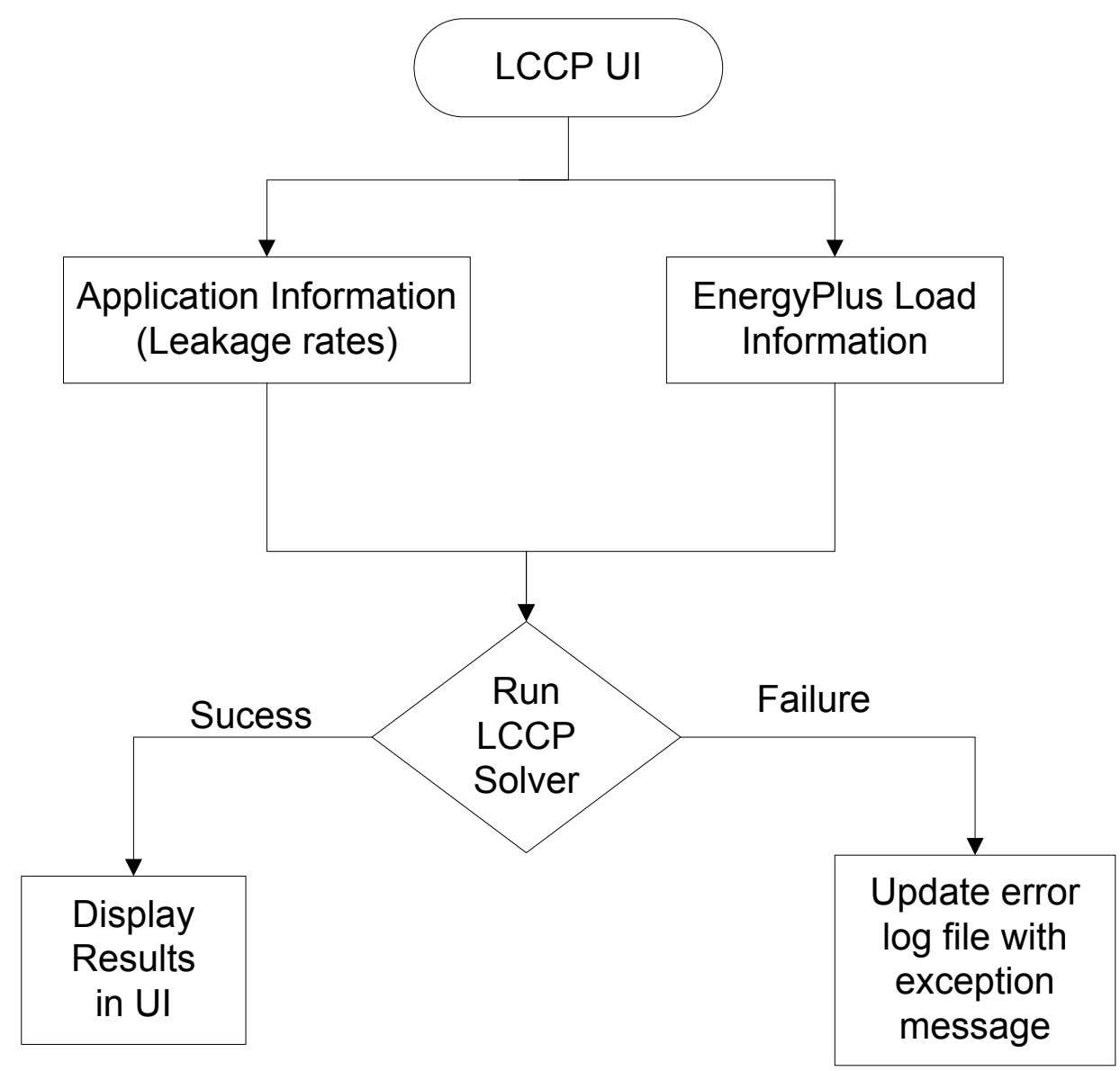

Figure 3: Flow Chart of the LCCP Desktop Application using Energy Plus for power consumption calculation.

The third option is to specify the hourly power consumption directly from the "Simulation Information" without requiring the "Load Information". This case is applicable to the "Display Cases Using Secondary Refrigerants" and the "Water Chiller" systems. The flow chart for this option is shown in Fig. 4 below. 


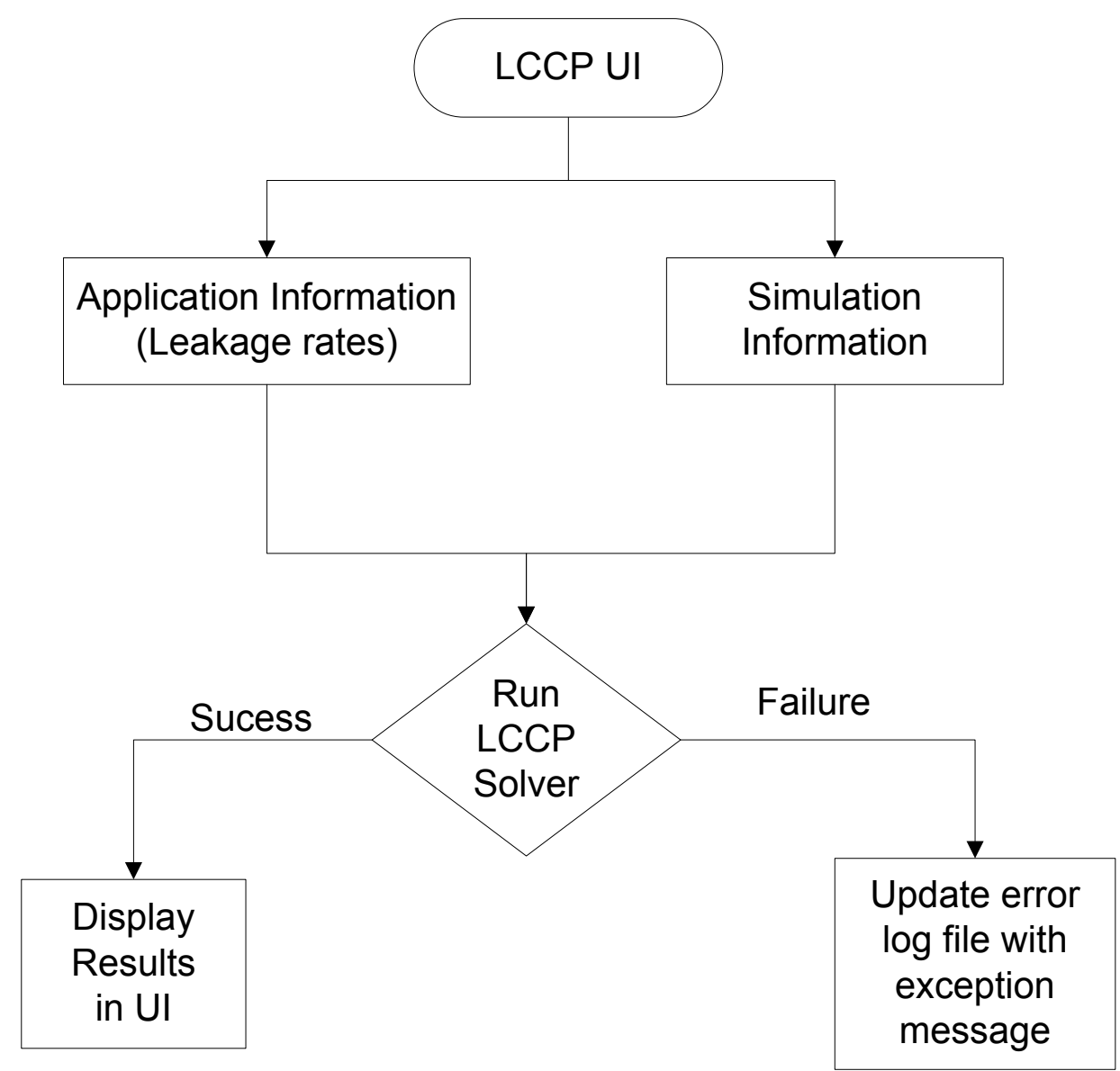

Figure 4: Flow Chart of the LCCP Desktop Application with no load information required.

\section{VapCyc Executable}

This section covers the use of VapCyc as the system simulation software. This is just an example to demonstrate how the system simulation software is used. Other system simulation software can be used with LCCP tool. It is also worth mentioning that VapCyc is not part of the open source license of the LCCP desktop tool.

The VapCyc executable requires the following three inputs: the path to the file containing the hourly load and weather data, the path to the VapCyc input file, and the path to the output file into which the output will be written.

Initially, the VapCyc executable reads the weather data and load data from the first input file. Then, the weather data is placed into bins. That is, the ambient temperatures are divided into ranges, or bins, of $10^{\circ} \mathrm{C}$ and the number of hours for which the temperature falls within each bin 
is determined. Subsequently, the three most frequent average bin temperatures (i.e., the bins which contain the most number of hours) are saved. Furthermore, three additional temperatures are saved: the maximum ambient temperature, the minimum ambient temperature, and the design temperature $(310.928 \mathrm{~K})$. These six temperatures are then used to perform the VapCyc simulations.

For every one of the six saved temperatures, the VapCyc executable loads the VapCyc input file (i.e., the input file which results from replacing the "Key" with the "value"), sets the condenser air temperature to the corresponding saved bin temperature, and runs the simulation. The results for the six runs, consisting of system capacity and coefficient of performance (COP), are saved and curve fitting is performed using a polynomial of the second degree to obtain the annual hourly power consumption of the system (calculated hourly capacity/calculated hourly COP).

The output file from the VapCyc executable is then written to a text file which is suitable to LCCP and contains the following six columns: hour, DBT, RH, COP, capacity, and power consumption.

\section{EnergyPlus}

When the user selects EnergyPlus from the "Load Type" drop down menu in the "Load Inputs", the user must choose from one of two options in a new drop down menu which appears. The two options are "Load" or "Power Consumption".

If the user chooses "Load", EnergyPlus will be used to obtain the hourly load profile (in Watts) as well as the weather data (DBT and RH) required for input to the system simulation software. On the other hand, by choosing "Power Consumption", EnergyPlus will be used to obtain the system's hourly power consumption (in kW-hr).

For both options, the LCCP software requires six more inputs from the user, five of which are required by the EnergyPlus software. The additional inputs are accessed by pressing the "..." button which appears to the right of the "Load Type" drop-down menu.

i. Path to the IDF file: The user browses and selects the input file containing the building and system information (.idf file). This file can be located anywhere on the user's 
computer. The user will be asked to locate it. Note that the folder containing the idf file will be the same folder where the EnergyPlus simulation output files must be placed (including the .csv file).

ii. Path to EnergyPlus install folder: The user browses and selects the folder in which EnergyPlus is installed. The default value for this input is C:|EnergyPlusV7-2-0 and is the default value for current installations of EnergyPlus.

iii. Path to weather file: The user browses and selects the EnergyPlus input weather data file (.epw) which must be located in the "WeatherData" folder located inside the "Energy Plus" installation directory (e.g. EnergyPlusV7-2-0 directory) on the user's computer (this is the default location for weather data files when installing Energy Plus).

iv. Working folder path: The user browses and selects the working folder for EnergyPlus (typically the folder containing the .idf file).

v. Command: The user enters the EnergyPlus input files which appear in the command line to execute EnergyPlus. The default value for this input is \%\%IDF_FILE\%\% $\% \%$ WEATHER_FILE\%\% and modification of this default value will typically not be necessary.

vi. Column header name: The LCCP software must be able to locate the desired data in the .csv output file of Energy Plus and read this data in order to perform the LCCP calculations. At this prompt, the user inputs the column header of the column containing the desired information to be read (i.e. load or power consumption). For instance, if the user wants to select the "Total Building Electric Demand" from the output list (when selecting the data which he wants to get in the output file) as the hourly system's power consumption, the output .csv file must have such a column with the header name "Whole Building:Total Building Electric Demand [W](Hourly)". Thus, the user will write "Whole Building:Total Building Electric Demand [W](Hourly)" in the column header input text box in the LCCP software. In order to avoid confusion or receiving errors, the user can obtain this header by running the Energy Plus simulation one time and copying the header of the required column and pasting it in the input text box in LCCP software. In the case of running a system which has multiple cycles, the user enters the desired column headers, separating each with a semicolon. 
It is worth mentioning that since the hourly data is required, when selecting the data to be reported in the EnergyPlus output from the IDF editor tool, the "reporting frequency" should be set to either "Hourly" or "Time Step" with the time step being set to 1 hour. Also, if EnergyPlus is used for load calculation rather than power consumption calculation, the DBT and the RH of the outdoor air should be selected when the user is selecting the data to be reported in the EnergyPlus output from the IDF editor tool.

Finally, the user should ensure that the number of cycles in the system selected in the "System Inputs" should be equal to the number of cycles in EnergyPlus. 


\section{Part 3: Interfaces}

\section{LCCP interfaces}

The LCCP code has 4 different interfaces:

1. ILCCPApplicationInfo

2. ILCCPSystem

3. ILCCPSolver

4. ILCCPResults

The "ILCCPApplicationInfo" is responsible for the "Application Information" inputs which are explained in section 5. This interface has 1 Bolean check, and 3 main methods in it. A summary of these methods is shown in Table 2. The Boolean check is "Initialize" which is true if application information is initialized properly. The first method is the "GetUnitPowerPlantEmissions" method which returns the hourly Emissions per unit Energy for the location. The other method is the "GetLoadValues" which is responsible for returning the hourly DBT, dew point temperature, RH, and absolute load values (in W) to the LCCP solver. The third method is the "GetEnergyConsumption" which returns the actual hourly energy consumption for each cycle in the system. Currently, the ILCCPApplicationInfo is implemented in 3 different classes in the distributed version of the LCCP desktop software. The first class is the "LCCPApplicationBase" class for built-in load type (see section 6.1.1) and file based load type (see section 6.1.2), while the second one is the "EPApplicationInfo" for the EnergyPlus load type (see sections 6.1.3 and 10), and the third one is the "ASHPApplicationInfo" for the AHRI Standard load type (see section 6.1.4). Each of the first two classes initialize the LCCP application (reads the TMY\# weather file, TMY\# emissions data file, and the load values .csv file), sets the required inputs for load calculation, and outputs the weather data and load values. In addition, this interface has 2 methods for saving and loading the application information xml node in the lccp file. 
Table 2: List of methods in the ILCCPApplicationInfo interface.

\begin{tabular}{|l|l|}
\hline \multicolumn{1}{|c|}{ Method } & \multicolumn{1}{|c|}{ Description } \\
\hline GetUnitPowerPlantEmissions & returns the hourly Emissions per unit Energy for the location \\
\hline GetLoadValues & $\begin{array}{l}\text { returns the hourly DBT, dew point temperature, RH, and } \\
\text { absolute load values (in W) to the LCCP solver }\end{array}$ \\
\hline Initialize & returns true if application information is initialized properly \\
\hline GetEnergyConsumption & $\begin{array}{l}\text { returns the actual hourly energy consumption for each cycle in } \\
\text { the system }\end{array}$ \\
\hline SaveToXml & saves the application information xml node in the lccp file \\
\hline LoadFromXml & loads the application information xml node in the lccp file \\
\hline
\end{tabular}

The "ILCCPSystem" has one main method (void GetHourlyPerformance(double[] dbt, double[] dpt, double[] rh, out double[,] capacity, out double[,] power)). This method is responsible for getting the hourly power consumption value from the cycle. This can be either directly (if power consumptions value load type is selected), or through running a system simulation tool (e.g. VapCyc). For VapCyc (or any other system simulation software that the user might want to use with the LCCP desktop application), the VapCyc is called (based on the required VapCyc inputs obtained from the UI) and the values obtained from it (power consumption values) inside this method. A summary of this method is shown in Table 2. Also, this interface has various methods to report the direct emissions (Dictionary<string, double> GetSecondaryDirectEmissions()), the indirect emissions (Dictionary<string, double> GetSecondaryInDirectEmissions()), results table (Dictionary<string, ResultsDataTable> GetResultTables()). In addition, this interface has 2 methods for saving and loading the application information xml node in the lccp file. 
Table 3: List of methods in the ILCCPSystem interface.

\begin{tabular}{|l|l|}
\hline \multicolumn{1}{|c|}{ Method } & \multicolumn{1}{|c|}{ Description } \\
\hline GetHourlyPerformance & returns the hourly power consumption value from the cycle \\
\hline GetSecondaryDirectEmissions & $\begin{array}{l}\text { Reports the named direct emissions calculated as a part of the } \\
\text { system }\end{array}$ \\
\hline GetSecondaryInDirectEmissions & $\begin{array}{l}\text { Reports the named indirect emissions calculated as a part of } \\
\text { the system }\end{array}$ \\
\hline GetResultTables & Reports the results table \\
\hline SaveToXml & saves the simulation information xml node in the lccp file \\
\hline LoadFromXml & loads the simulation information xml node in the lccp file \\
\hline
\end{tabular}

The "ILCCPSolver" has two methods methods. The first one has a Boolean return (bool SetLCCPInputs(ILCCPSystem system, ILCCPApplicationInfo appInfo, BasicSystemInputs inputs)). This method is responsible for changing the basic system inputs which are received from the user through the UI to LCCP inputs (in the form of application and system inputs). The basic system inputs (from the "BasicSystemInputs" class) contain the city name "City", system life time "LifetimeYrs", number of cycles in the system "CycleCount", different emission inputs "emmInputs" ( $\mathrm{CO}_{2}$ equivalent and GWP), and the different cycle inputs "inputs" (from the "CycleInputs" class). The cycle inputs include the system type, refrigerant name, charge, nominal load, different leakage rates, and the mass of the different materials in the system. The main class which implements this interface is the "LCCPSolver" class. This class contains a method "CalculateIndirectFromEnergy" which obtains the hourly performance (from the "GetLoadValues" and the "GetHourlyPerformance" methods) and then obtains the hourly energy consumption of the cycle (based on its refrigeration capacity and power consumption required). The different cycle emissions (i.e. LCCP core calculation for direct and indirect emissions) are 
performed in this class. The second method is the Run method which returns the LCCP results. A summary of these 2 method is shown in Table 3.

Table 4: List of methods in the ILCCPSolver interface.

\begin{tabular}{|l|l|}
\hline \multicolumn{1}{|c|}{ Method } & \multicolumn{1}{c|}{ Description } \\
\hline SetLCCPInputs & $\begin{array}{l}\text { changes the basic system inputs which are } \\
\text { received from the user through the UI to LCCP } \\
\text { inputs }\end{array}$ \\
\hline Run & returns the LCCP results \\
\hline
\end{tabular}

The "ILCCPResults" has checks for the success of the run of the LCCP software. A summary of these methods is shown in Table 4. This interface is implemented by the "LCCPResults" class. This class is mainly responsible for summing up the different emissions (direct and indirect) of the cycles in the system to obtain the system emissions.

Table 5: List of methods in the ILCCPResults interface.

\begin{tabular}{|l|l|}
\hline \multicolumn{1}{|c|}{ Method } & \multicolumn{1}{c|}{ Description } \\
\hline ErrorCode & returns 0 if no error is detected \\
\hline ErrorMessage & Sets the error message if any error is detected \\
\hline SetError & $\begin{array}{l}\text { Sets the error code and message if any error is } \\
\text { detected }\end{array}$ \\
\hline Success & returns true if LCCP calculation is successful \\
\hline
\end{tabular}




\section{References}

ANSI/AHRI, 2011. ANSI/AHRI 1320-2011, Performance Rating of Commercial Refrigerated Display Merchandisers and Storage Cabinets for Use With Secondary Refrigerants.

ANSI/AHRI, 2011. ANSI/AHRI 550/590-2011 with Addendum 1, Performance Rating Of Water-Chilling and Heat Pump Water-Heating Packages Using the Vapor Compression Cycle.

ANSI/AHRI, 2008. ANSI/AHRI 210/240-2008 with Addenda 1 and 2, Performance Rating of Unitary Air-Conditioning \& Air-Source Heat Pump Equipment.

Deru, M., and Torcellini, P. 2007. Source Energy and Emission Factors for Energy Use in Buildings. NREL Technical Report NREL/TP-550-38617. National Renewable Energy Laboratory: Golden, CO.

Hwang, Y., Jin, D.-H., and Radermacher, R. 2007. Comparison of R-290 and two HFC blends for walk-in refrigeration systems. International Journal of Refrigeration 30(4): 633-641.

NREL. 2012a. National Solar Radiation Data Base, 1991-2005 Update: Typical Meteorological Year 3. Renewable Resource Data Center, National Renewable Energy Laboratory: Golden, CO. Available at: http://rredc.nrel.gov/solar/old_data/nsrdb/19912005/tmy3/by_state_and_city.html

NREL. 2012b. U.S. Life Cycle Inventory Database. National Renewable Energy Laboratory: Golden, CO. Available at: http://www.nrel.gov/lci/

Papasavva, S., Hill, W.R., and Andersen, S.O. 2010. GREEN-MAC-LCCP: A Tool for Assessing the Life Cycle Climate Performance of MAC Systems. Environmental Science \& Technology 44(19): 7666-7672.

Sand, J.R., Fischer, S.K., and Baxter, V.D. 1997. Energy and Global Warming Impacts of HFC Refrigerants and Emerging Technologies: TEWI-III. Presented at the International Conference on Climate Change, Baltimore, MD, 12-13 June 1997. 